\title{
LA EPIDEMIA DE LA OBESIDAD EN ESPAÑA: LA RETÓRICA DE SU DISCURSO Y RIESGOS POTENCIALES
}

\author{
A EPIDEMIA DE OBESIDADE NA ESPANHA: A RETÓRICA DO SEU DISCURSO \\ E RISCOS POTENCIAIS
}
THE OBESITY EPIDEMIC IN SPAIN: THE RHETORIC OF ITS DISCOURSE AND POTENTIAL RISKS

Miguel Company-Morales*, Alvaro Sicilia-Camacho**, María Filomena Rubio-Jiménez ${ }^{\star \star \star}$, Andrés Fontalba-Navas ${ }^{\star \star \star \star}$

\begin{abstract}
Palabras clave Obesidad.

Epidemia.

Estereotipo.

Promoción de la

salud.

Resumen: La obesidad se ha convertido en una prioridad para las políticas de salud pública en España. Basándose en el aumento de su prevalencia ha sido catalogada como una epidemia. A pesar de estas afirmaciones, las investigaciones no parecen siempre tan concluyentes. El objetivo de este artículo es revisar algunas de las 'verdades' en torno a la obesidad: a) que la obesidad va en aumento y es una epidemia en España; b) que existe una relación directa entre peso y salud; y c) que la obesidad tenga su consecuencia única en el desequilibrio entre gasto y consumo energético. Además, dado que las ideas vertidas en torno a la epidemia de la obesidad, y el modo exagerado y alarmista en que se produce, pueden tener consecuencias dañinas e impredecibles sobre la salud de las personas, un segundo objetivo es delinear algunos de los posibles riesgos de estas prácticas de comunicación.
\end{abstract}

Palavras-chave Obesidade.

Epidemia.

Estereotipagem.

Promoção da saúde.

Keywords

Obesity.

Epidemic.

Stereotyping.

Health promotion.
Resumo: A obesidade tornou-se uma prioridade para as políticas de saúde pública na Espanha. Com base no aumento da prevalência foi classificada como uma epidemia. Apesar dessas afirmações, as investigações nem sempre parecem tão conclusivas. O objetivo deste artigo é analisar algumas das 'verdades' sobre a obesidade: a) que a obesidade está aumentando e é uma epidemia em Espanha; b) existe uma relação direta entre peso e saúde; e c) que a obesidade tenha uma consequência única em um desequilíbrio entre gasto e consumo de energia. Além disso, uma vez expressas as ideias sobre a epidemia da obesidade, a maneira exagerada e alarmista que ocorre pode ter consequências imprevisíveis sobre a saúde das pessoas; um segundo objetivo é delinear alguns dos possíveis riscos dessas práticas de comunicação.

Abstract: Obesity has become a top priority for public health policies in Spain. Based on increasing prevalence, it has been classified as an epidemic. Despite these claims, research does not always seem so conclusive. The purpose of this article is to review some of the 'truths' about obesity: a) that it is on the rise and is an epidemic in Spain; b) that there is a direct relationship between weight and health; c) that its sole effect is the imbalance between energy expenditure and intake. In addition, since the ideas expressed about the obesity epidemic and the exaggerated and alarmist way they are produced might have harmful and unpredictable consequences on people's health, a second aim is to point out some of the possible risks inherent in those communication practices.
* Universidad de Almería. Almería, Espanha.

E-mail: miguelcompanymorales@gmail. com

** Universidad de Almería. Almería, Espanha.

E-mail: asicilia@ual.es

*** Servicio Andaluz de Salud. Área de Gestión Sanitaria Norte de Almería, Espanha.

E-mail: marienarubiojimenez@gmail.com

**** Universidad de Almería. Almería, Espanha.

E-mail: andres.fontalba.sspa@ juntadeandalucia.es

Recebido em: 09-11-2015 Aprovado em: 25-06-2016

(c) (1) (8) Licence 


\section{INTRODUCCIÓN: LA RETÓRICA SOBRE LA EPIDEMIA DE LA OBESIDAD}

En los últimos años la obesidad se ha convertido en una prioridad para las políticas de salud pública. Basándose en el aumento de su prevalencia, la obesidad ha sido catalogada como una epidemia en la sociedad occidental. Algunos autores han llegado a catalogar la obesidad como la epidemia del siglo XXI (MORENO; MONEREO; ÁLVAREZ, 2000). La Organización Mundial de la Salud (en adelante OMS) y la Comisión Europea aseguran que el sobrepeso y la obesidad reducen la esperanza de vida, debido a las complicaciones metabólicas y cardiovasculares que producen (COMISIÓN EUROPEA, 2007; OMS, 2004) Las instituciones sanitarias españolas también muestran una gran preocupación con los temas relacionados con la salud y la obesidad.

Durante las últimas décadas los medios de comunicación no han dejado también de hacerse eco de esta preocupación de una forma alarmista. Recientemente, el diario El País, publicaba un artículo cuyo título reza "Europa se enfrenta a una epidemia de obesidad en 2030" (SEVILLANO, 2015). En él se advierte que Europa se enfrenta a una crisis de obesidad "de enormes proporciones". Esta noticia supuestamente se basa en evidencias científicas extraídas de diferentes fuentes, entre las que se menciona, las proyecciones presentadas por la oficina regional europea de la OMS, el Congreso Europeo de Obesidad celebrado en mayo 2015 en Praga, o un monográfico sobre obesidad publicado en el mes de febrero en la prestigiosa revista The Lancet. El diario El Mundo se hace eco también de esta noticia y resalta que en España estas cifras serán similares con un $70 \%$ de personas con sobrepeso y un 30 $\%$ obesidad (LÓPEZ, 2015).

A pesar de estas dramáticas noticias, los resultados de las investigaciones no parecen siempre tan concluyentes. Los datos nacionales e internacionales recientes no parecen indicar nada nuevo respecto al siglo XX. De hecho, algunos trabajos han resaltado que el gran cambio en el discurso de la obesidad ha sido su retórica (CAMPOS, 2004; GARD, 2011; GARD; WRIGHT, 2005; OLIVER, 2006). Oliver (2006) ha denunciado la forma exagerada en que se ha informado sobre la obesidad y su predicción. De hecho, se han llegado a dar cifras desorbitadas de que la obesidad producía entre 300 y 400 mil muertes de norte americanos por año, cuando en realidad no es posible conocer el número de muertes relacionadas directamente con la obesidad. En esta misma línea, Gard y Wright (2005) compararon la retórica que utiliza la ciencia moderna en torno al discurso de la obesidad y aquel utilizado por los medios de comunicación (GARD; WRIGHT, 2005). En su comparación los autores manifiestan su sorpresa ante el modo hiperbólico del lenguaje que utilizan algunos científicos y cómo algunas de las opiniones y predicciones sobre la obesidad han sido formadas por exageraciones.

Ante las alarmas que diferentes instituciones y medios de comunicación han creado en torno a la epidemia de la obesidad, parece prudente revisar algunos de estos reclamos de acuerdo con las evidencias científicas. En este sentido, el presente artículo tiene un doble objetivo. Por un lado, el artículo pretende mostrar que al menos tres de los principales reclamos realizados en torno a la obesidad mantienen ciertas incertidumbres. En concreto, como será defendido en los apartados siguientes: a) existen incertidumbres sobre que la obesidad vaya en aumento y sea una epidemia en España; $b$ ) no es demostrable que exista una relación directa entre peso y salud; $y$ c) no es cierto que el sobrepeso y la obesidad sea una consecuencia únicamente del desequilibrio entre gasto y consumo energético. Por otro lado, dado que 
las ideas vertidas en torno a la epidemia de la obesidad, y sobre todo el modo exagerado y alarmista en que se producen, pueden tener consecuencias dañinas e impredecibles sobre las personas, un segundo objetivo es delinear algunos de los posibles riesgos de estas prácticas de comunicación. Con ello se pretende advertir que el lenguaje no es neutro, y que el modo en que la obesidad es construida en la opinión pública puede tener también efectos no deseados en la población.

\section{REVISANDO ALGUNAS EVIDENCIAS SOBRE LA EPIDEMIA DE LA OBESIDAD}

Académicos, instituciones y medios de comunicación reiteradamente han advertido de las consecuencias de la epidemia de la obesidad en nuestra sociedad (AGENCIA ESPAÑOLA DE SEGURIDAD ALIMENTARIA Y CONSUMO, 2013; 2014; HERNÁNDEZ et al., 1998; LÓPEZ, 2015; OMS, 2004; 2015; SEVILLANO, 2015). No obstante, una revisión en profundidad de los datos y las investigaciones realizadas sobre el tema advierten que muchos de los reclamos realizados no tienen suficiente evidencia científica. Además, las revisiones realizadas hasta el momento han destacado que existen incertidumbres que no han trascendido a los ciudadanos con la misma fuerza que las certezas (CASAZZA et al., 2013). En este artículo revisamos las evidencias existentes de acuerdo a tres serios e importantes reclamos recogidos en el discurso sobre la obesidad.

\section{CERTEZA 1: LA OBESIDAD ES LA EPIDEMIA DEL SIGLO XXI}

Las autoridades sanitarias afirman que estamos inmersos en una "epidemia" mundial de obesidad debido al crecimiento exponencial de peso de las personas (COMISIÓN EUROPEA, 2007). Según la Agencia Española de Seguridad Alimentaria y Consumo, (en adelante AESA), "la prevalencia de la obesidad y su tendencia ascendente durante las dos últimas décadas, han hecho que también se afiance en España el término de obesidad epidémica" (AESA, 2005, p.10). Algunas investigaciones realizadas en España, como el "estudio ENRICA", han afirmado recientemente que "a menos que la epidemia de obesidad sea controlada [...] podrían verse amenazados los logros alcanzados en las últimas décadas en el control de la enfermedad coronaria en España" (GUALLAR-CASTILLÓN et al., 2014, p. 373). Del mismo modo, en las conclusiones de la Encuesta Nacional de Salud (en adelante ENS) (2012) se afirma que "continúa aumentando la obesidad", y se indica que "desde la primera ENS en 1987, la obesidad sigue una línea ascendente en ambos sexos, más marcada en hombres que en mujeres" (MINISTERIO DE SANIDAD, SERVICIOS SOCIALES E IGUALDAD, 2013). Lógicamente noticias en los medios de comunicación han abordado con un tono alarmante lo que han considerado un serio problema de salud pública (LÓPEZ, 2015; SEVILLANO, 2015).

No obstante, una revisión de la investigación reciente en España no parece apoyar este reclamo en la actualidad. El aumento del sobrepeso y obesidad en España podría haberse producido desde 1987(39,7\%) hasta 2006 (53,3\%) según la ENS (MINISTERIO DE SANIDAD, SERVICIOS SOCIALES E IGUALDAD, 2007). Sin embargo, la última ENS, elaborada en el año 2012, indica una prevalencia de sobrepeso y obesidad en la población adulta del 53,7\% (MINISTERIO DE SANIDAD, SERVICIOS SOCIALES E IGUALDAD, 2013). Con estos datos podemos afirmar que en la actualidad estamos en una situación de estancamiento, pues el incremento total de la prevalencia de sobrepeso y obesidad en el periodo transcurrido entre 
las dos últimas ENS (2006-2012) ha sido sólo del 0,4\% (ver figura 1). Además, los datos sobre obesidad y sobrepeso en población adulta señalada en España a través de la última ENS coinciden con los datos de la encuesta europea de salud (INSTITUTO NACIONAL DE ESTADÍSTICA, 2009), que había indicado también una prevalencia del 53,7 \% .

Figura 1 - Prevalencia de sobrepeso y obesidad en mayores de 18 años según las ENS 2006-2012

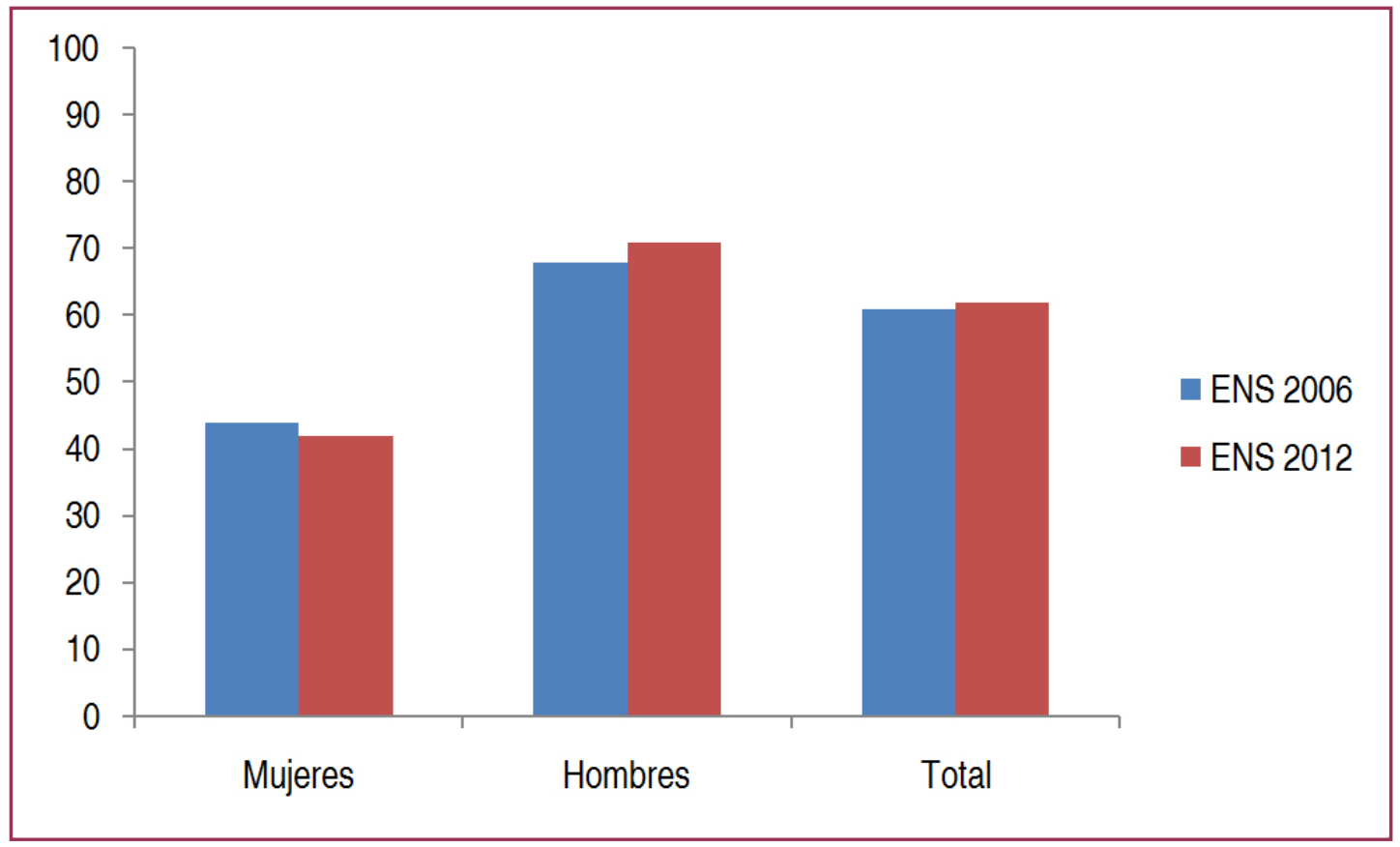

Fuente: Elaboración propia a partir de los datos de las ENS 2006 y 2012

Los datos sobre población adulta no reflejan un crecimiento alarmante del sobrepeso y obesidad pero, ¿qué ocurre con la población infantil? Los criterios para indicar que un niño o niña tienen obesidad infantil han variado en los últimos años. A finales del siglo pasado se utilizó una definición de obesidad infantil basada en percentiles poblacionales (locales) del Índice de Masa Corporal (en adelante IMC), de tal modo que la obesidad se localizaba por encima de los valores del percentil 95 para la edad y el sexo. Basándose en estos criterios, en España se utilizaron desde el año 1988 las tablas de la Fundación Orbegozo (HERNÁNDEZ et al., 1998), resultando investigaciones como el estudio EnKid (1998-2000), que observó una prevalencia de sobrepeso y obesidad del $30,4 \%$ en niños y niñas entre 6 y 9 años (SERRA et al., 2003). Sin embargo, esta opción pronto mostró algunas limitaciones, debido a que las tablas disponibles de valores de IMC poblacionales reflejan la distribución gaussiana (normal) de una población. Diferentes propuestas han sido presentadas hasta la fecha. La OMS propuso utilizar los percentiles 85 y 95 de las tablas de los Centers for Disease Control and Prevention (CDC). Por otra parte, un comité de la International Obesity Task Force (IOTF) decidió adoptar los puntos de corte de IMC establecidos por Cole et al. (2000), elaborados mediante una proyección matemática de los valores correspondientes a la definición para los adultos de obesidad (IMC >30 kg/m2) y sobrepeso (IMC>25 kg/m2).

De uno u otro modo, los investigadores han dispuesto de diferentes criterios de referencia a la hora de establecer y clasificar a la población infantil respecto a su IMC. En España los estudios EnKid (1998-2000) y ALADINO (2010-2011) (AESA, 2011) son el paradigma de estas diferencias de criterio en la comparación de tablas de referencia para el IMC en niños. De 
hecho, mientras que el estudio Enkid realizó su clasificación de la población infantil respecto al IMC teniendo en cuenta las tablas de la fundación Obergozo (HERNÁNDEZ et al., 1998), el estudio ALADINO (2010-2011) toma como referencia los criterios establecidos por Cole et al (2000). Con ello una comparación a primera vista de los resultados de ambos estudios nos llevaría a conclusiones erróneas. Por ejemplo, el estudio ALADINO informa de una prevalencia de sobrepeso y obesidad infantil del $44,5 \%$ en niños y niñas entre 6 y 9 años cuando compara sus resultados directamente con el estudio Enkid. Sin embargo, cuando los mismos resultados del estudio ALADINO (2010-2011) son comparados considerando como referencia las tablas de la Fundación Orbegozo, la prevalencia de sobrepeso y obesidad baja al 30,8\% para la misma población. Por lo tanto, si la comparación de estudios se realiza considerando los mismos criterios de referencia se puede afirmar que la prevalencia de sobrepeso y obesidad infantil no ha aumentado en España en el periodo de once años que abarcan ambos estudios (ver figura 2).

Figura 2 - Comparación prevalencia sobrepeso y obesidad en los estudios EnKid y ALADINO

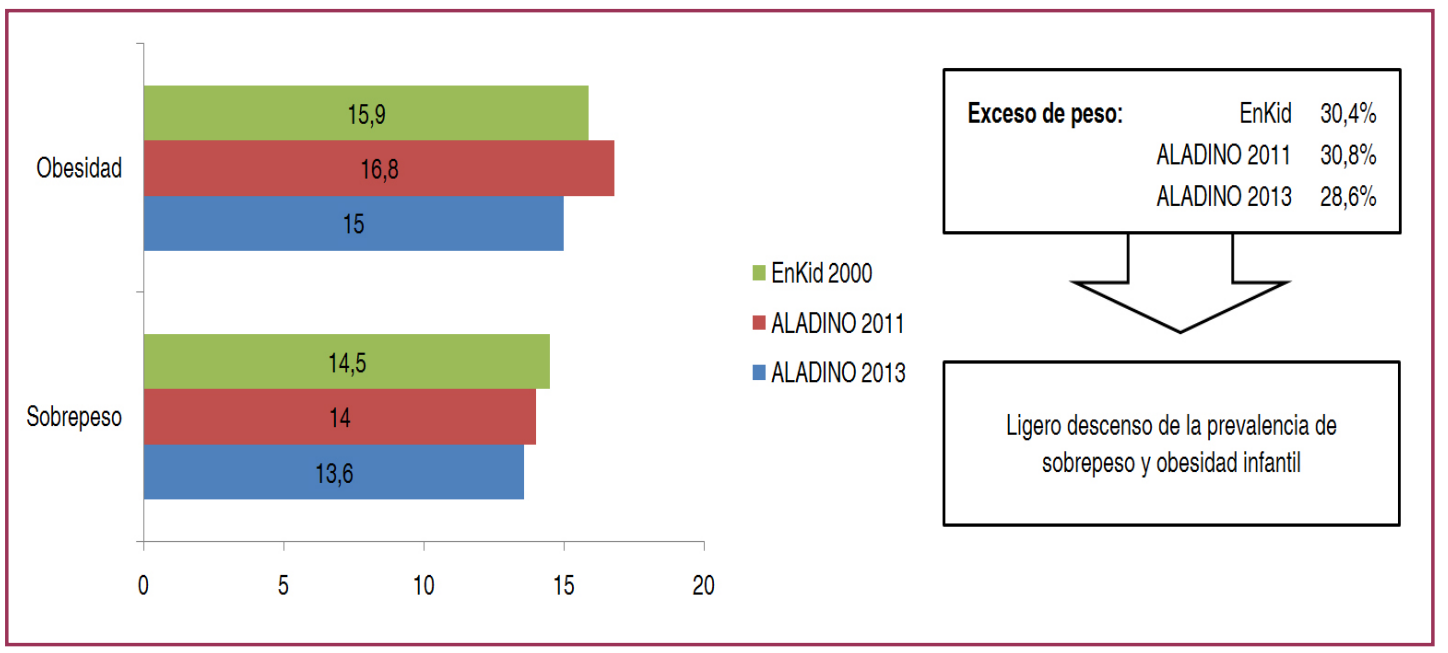

Fuente: Elaboración propia a partir de los datos de la Agencia Española de Seguridad Alimentaria y Consumo

La AESA ha confirmado que no existe un aumento del sobrepeso y obesidad en población infantil con un nuevo estudio "ALADINO" en el año 2013. Esta evidencia ha sido también confirmada a través de los resultados de la ENS 2012, donde se indica que la prevalencia de sobrepeso y obesidad en niños y niñas entre 2-17 años es del $27,7 \%$, produciéndose un estancamiento con respecto a la ENS $2006(27,6 \%)$. Por lo tanto, los recientes resultados de las investigaciones realizadas en España no apoyan los reclamos de que el sobrepeso y la obesidad vayan aumentando de forma alarmante. Más bien los resultados indican que se ha producido un estancamiento, o incluso una recesión, de la prevalencia del sobrepeso y obesidad en línea con lo que parece estar sucediendo en el resto de países occidentales (OGDEN et al., 2014)

\section{CERTEZA 2: EXISTE UNA RELACIÓN DIRECTA ENTRE EL PESO Y LOS PROBLEMAS DE SALUD}

Investigadores, autoridades sanitarias y medios de comunicación nos alertan del peligro que supone para las personas poseer un IMC superior al límite normal $\left(25 \mathrm{Kg} / \mathrm{m}^{2}\right)$. La OMS advierte que pasar este nivel de IMC supone un importante factor de riesgo de enfermedades 
cardiovasculares, diabetes, trastornos del aparato locomotor y algunos cánceres (especialmente de endometrio, mama y colon) (OMS, 2015) .

En todas estas advertencias, se establece una relación directa entre aumento de peso y ciertas enfermedades. Sin embargo, una revisión de la literatura científica nos sugiere que esta relación no es tan clara y directa. Flegal y colaboradores han analizado la asociación entre sobrepeso y obesidad con las enfermedades cardiovasculares y cáncer (FLEGAL et al., 2005; 2007). Por un lado, los resultados de los estudio de Flegal y colaboradores (2005) han evidenciado que la mortalidad parece asociada más con la obesidad y no tanto con el sobrepeso. Respecto a la relación entre peso y mortalidad por cáncer, Flegal y colaboradores (2007) ponen de manifiesto que globalmente no existe ninguna relación entre aumento de mortalidad y nivel de IMC. En un estudio más reciente, Flegal y colaboradores (2013), han vuelto a investigar la relación entre IMC y mortalidad. Tras una revisión sistemática y meta-análisis de estudios que relacionan el IMC y las causas de mortalidad, los autores concluyen que sólo determinados grupos de obesidad mostraron relación con mayor mortalidad. En concreto, sólo las personas con obesidad grado II y III se asociaron a una mayor posibilidad de mortalidad en relación con las personas con peso normal. Así, las personas con obesidad grado I no se asociaron con mayor mortalidad, e incluso los grupos sobrepeso mostraron una mortalidad significativamente menor con respecto a las personas con bajo peso.

Flegal y colaboradores (2007) coinciden con otros investigadores. O'Brien y colaboradores (2006) y Romero-Corral y colaboradores (2006) concluyen en sus estudios que los pacientes con sobrepeso presentan menor mortalidad por causas cardiacas y cáncer que en el resto de grupos. En estos estudios sólo se ha encontrado una asociación entre el grupo de obesos tipo II y III y mortalidad por enfermedad cardiaca, no detectándose esta asociación para los obesos tipo I. En la misma línea, Oreopoulos y colaboradores (2008) sugieren que los enfermos con insuficiencia cardiaca crónica con sobrepeso u obesidad presentan un índice de mortalidad menor que los enfermos con bajo peso o peso normal.

Por lo tanto, los estudios internacionales muestran que existen débiles evidencias entre el aumento del peso corporal medido por el IMC y el aumento potencial de padecer una enfermedad grave o mortal. Todavía se conoce muy poco por qué las personas con bajo peso y las personas muy obesas $\left(\mathrm{IMC}>35 \mathrm{Kg} / \mathrm{m}^{2}\right)$ tienen más probabilidades de morir que las personas con peso "normal". Sin embargo, parece más claro que la relación entre el aumento de la mortalidad conforme aumenta el índice de masa corporal debe ser objeto de un mayor número de estudios en busca de evidencias sobre cuál es el rango de adiposidad que protege la salud.

Los estudios internacionales indicados más arriba han supuesto en los últimos años una motivación para la investigación en materia de obesidad y una mayor cautela a la hora de establecer una asociación directa y lineal entre IMC y determinadas enfermedades. Con la aparición de nuevos estudios se empieza a refutar las creencias que señalan al acumulo de grasa como un factor de riesgo para la salud en sí mismo.

Por otro lado, grupos de investigadores, que inicialmente se habían sumado a la defensa de la relación directa entre IMC y determinadas enfermedades, parecen moderar e incluso alterar su discurso a la luz de nuevas evidencias. Así, por ejemplo, Ortega y colaboradores (2013) llegan a afirmar que obesidad y salud no están reñidas. En el estudio longitudinal realizado con 43.265 participantes estos autores analizan la relación entre el porcentaje de grasa corporal 
y la aptitud física. El 29,7\% de los participantes del estudio fue calificado con obesidad y, de éstos, casi la mitad fueron considerados "metabólicamente saludables". En estas personas los niveles de presión arterial, triglicéridos, colesterol y glucemia basal estaban dentro de los parámetros considerados como normales. En este estudio los participantes metabólicamente saludables pero con obesidad tenían un menor riesgo a morir (30-50\%) que sus compañeros obesos y metabólicamente no saludables. Además, no existían diferencias de riesgo entre las personas metabólicamente saludables con obesidad y los participantes metabólicamente saludables con peso normal. La clave según los investigadores se encuentra en la realización de actividad física.

Ante las nuevas evidencias que parecen ir encontrándose, la Sociedad Española para el Estudio de la Obesidad (SEEDO) llamaba a la reflexión sobre la relación tan directa que ha sido establecida entre IMC y ciertas enfermedades, al mismo tiempo que advertía sobre el potencial efecto protector del sobrepeso para la salud (FOZ, 2008). En este sentido, esta institución reconocía que las nuevas evidencias sobre la relación entre sobrepeso y mortalidad tendrán que ser tenidas en cuenta cuando se elaboren nuevas guías de salud.

\section{CERTEZA 3: LA OBESIDAD ES CONSECUENCIA DEL DESEQUILIBRIO ENTRE EL CONSUMO Y LA INGESTA DE CALORÍAS}

La obesidad está definida por la OMS como una enfermedad crónica (OMS, 2004). Aunque no se conocen completamente los mecanismos que producen la obesidad se piensa que la mayoría son debidos a la interacción entre factores individuales (genéticos y metabólicos) y factores relacionados con el modo de vida (factores medioambientales). De hecho, la OMS señala principalmente a los factores medioambientales, particularmente a la alimentación y el sedentarismo, como el origen de la obesidad (COMISIÓN EUROPEA, 2007). Básicamente la explicación se simplifica de tal forma que se considera que las personas aumentan de peso al ingerir una cantidad de alimentos que aportan una energía superior a la consumida por su actividad física y metabólica En una nota descriptiva publicada en enero de 2015, la OMS intenta responder algunas cuestiones entorno al sobrepeso y obesidad. Ante la pregunta ¿Qué causa el sobrepeso y la obesidad?, la OMS llega a afirmar:

\footnotetext{
La causa fundamental del sobrepeso y la obesidad es un desequilibrio energético entre calorías consumidas y gastadas. En el mundo, se ha producido: a) un aumento en la ingesta de alimentos hipercalóricos que son ricos en grasa, sal y azúcares pero pobres en vitaminas, minerales y otros micronutrientes, y b) un descenso en la actividad física como resultado de la naturaleza cada vez más sedentaria de muchas formas de trabajo, de los nuevos modos de desplazamiento y de una creciente urbanización (OMS, 2015, p. 2).
}

A pesar de que el discurso sobre la obesidad pone el acento en la alimentación y el sedentarismo como causas principales, hoy día se conoce que existen otros factores implicados en el aumento de la adiposidad en las personas. De hecho, investigaciones recientes ponen de manifiesto el carácter multifactorial en el origen de la obesidad (BURT et al., 2014; CORRELLA; PORTOLÉS, 2008; HARSCH et al., 2003; JARRIN; MCGRATH; POIRIER, 2015). Coleman (2010) ha sugerido que la causa principal del aumento de peso de una persona es una química cerebral alterada. La leptina es la hormona producida por la grasa que actúa sobre el hipotálamo para regular la ingesta de alimentos, el gasto energético y la cantidad de grasa que se acumula, 
de tal manera que estos autores (COLEMAN, 2010; USHMA, 2010) han evidenciado que si falta la leptina en el organismo, o existe déficit en sus receptores, los mecanismos para acumular grasa se ven favorecidos. De este modo, Coleman destaca el papel regulador de la grasa sobre la ingesta de alimentos. En esta línea, Farooqi y O'Rahilly (2005) atribuyen la obesidad directamente a una mutación genética del receptor MC4 de leptina. Estos investigadores han encontrado familias con obesidad mórbida causada por una mutación del receptor MC4, considerando que esta mutación es la causa genética más frecuente de obesidad mórbida. Otros estudios han destacado la importancia de la cantidad y calidad del sueño en la regulación metabólica. Así, el sueño parece estar asociada a la regulación hormonal de la leptina y grelina, de tal manera que la alteración de los ritmos circadianos, especialmente en la infancia, podría favorecer la aparición del sobrepeso (JARRIN; MCGRATH; POIRIER, 2015). La consecuencia más importante de estas alteraciones neuroendocrinas es el aumento del apetito, especialmente por alimentos hipercalóricos y ricos en hidratos de carbono, para intentar sustituir la energía que no se recuperó por la falta de un sueño reparador (BURT et al., 2014).

En resumen, atribuir la causa del sobrepeso y obesidad al desequilibrio energético entre la ingesta y el gasto calórico supone un riesgo de simplificación que no refleja la complejidad mostrada por las evidencias científicas. Junto con el ambiente, factores genéticos y mecanismos hormonales y neurales intervienen también en la variación de peso (ALEMANY, 2003). Algunos de los factores causantes del sobrepeso y obesidad son claramente genéticos, aunque la investigación todavía intentan esclarecer la importancia que tiene la genética con respecto a los factores ambientales en la etiología de la obesidad (CORRELLA; PORTOLÉS, 2008). De hecho, la mayoría de personas con obesidad mórbida o extrema responden a esta causalidad multifactorial. Por lo tanto, no parece oportuno relacionar el aumento de la obesidad sólo con la profusión alimentaria característica de las sociedades modernas.

\section{COMENTARIOS FINALES. RIESGOS POTENCIALES DEL DISCURSO DE LA EPIDEMIA DE LA OBESIDAD}

En noviembre de 2009, el periódico la vanguardia (LUGILDE, 2009) se hacía eco de la intervención de la Xunta de Galicia para retirar la custodia de un niño con sobrepeso a unos padres de raza gitana. Diferentes razones como tener una familia desestructurada, dejadez en la alimentación, falta de ejercicio físico o irresponsabilidad de los padres fueron apuntadas como consecuencias de la obesidad en el niño. Más allá de la importante cuestión de la intervención de la administración para retirar a un chico del seno de su familia, el caso llama la atención por la reproducción de muchos de los mensajes que son utilizados en el discurso sobre la obesidad y que, en cierto modo, nos iluminan el modo en que se piensa y actúa sobre este asunto.

En las últimas décadas un gran número de informes de investigación, noticias, libros, artículos, programas de radio y televisión han apoyado de forma acrítica una campaña para 'salvar' a la población, y especialmente a los niños y niñas, del problema de la obesidad. El fenómeno de la obesidad es global y ha arrastrado una industria de miles de millones de euros en torno a dietas saludables, programas de ejercicio, gimnasios, programas de televisión, tratamientos, medicamentos contra la obesidad, etc. Sin embargo, salvo algunas excepciones (CAMPOS, 2004; EVANS; RICH; DAVIES, 2008; GARD; WRIGHT, 2005; HARWOOD, 2009), 
poco se ha parado a pensar sobre cómo estos mensajes están siendo recibidos e interpretados por los diferentes grupos de población y, especialmente, si estos mensajes pueden estar perjudicando la salud más que favorecer el bienestar de las personas.

Algunos investigadores sostienen que la vinculación de la obesidad con una "epidemia", la epidemia del siglo XXI, ha generado una situación de "pánico" y "crisis de la salud" en torno a la obesidad basada en el riesgo a enfermar (CAMPOS, 2004; EVANS et al., 2008; GARD; WRIGHT, 2005; HARWOOD, 2009). Esta aptitud y alarma, sin embargo, se muestra potencialmente peligrosa para la salud de los ciudadanos, sobretodo de los más jóvenes. Algunas investigaciones han mostrado el impacto drástico que los mensajes sobre la obesidad, y el ensalzamiento que un modelo de culto al cuerpo tiene en la población en general y en la población juvenil en especial (EVANS et al., 2008; GOÑI; RODRIGUEZ, 2004).

Por otro lado, los discursos públicos sobre la obesidad presentan una carga moral e ideológica muy acusada que fomentan la estigmatización de la persona con sobrepeso y obesidad (LÓPEZ DE LA TORRE; BELLIDO, 2008). Estar obeso es igual a estar enfermo. Las personas obesas son catalogadas como enfermos por el simple hecho de poseer un IMC superior a $30 \mathrm{Kg} / \mathrm{m}^{2}$, a pesar de que en la investigación no haya consenso sobre la relación directa del IMC y mortalidad (FLEGAL et al., 2005; 2007; 2013; O'BRIEN et al., 2006; OREOPOULOS, 2008; ROMERO-CORRAL et al., 2006). Nosotros entendemos que esta estigmatización es producto de una compresión limitada, insuficiente y/o parcial de la obesidad, la alimentación, la sociedad y la cultura.

Al tratar social y sanitariamente el problema de la obesidad como una epidemia se ha optado por generalizar acerca de las causas y las consecuencias de estar obeso y ello conlleva ciertas dificultades. Dado que la causa básica del sobrepeso y obesidad se achaca a un desequilibrio entre la ingesta de alimentos (regulado por la dieta) y el consumo de calorías (regulado por el gasto energético que produce la persona), la responsabilidad de tener exceso de peso queda fundamentalmente centrada en el individuo. Con ello existe una carga de moralidad en los discursos sobre la salud y la obesidad que atribuye una falta de disciplina y responsabilidad a las personas (o sus familias) con sobrepeso y obesidad. En otras palabras, asumiendo la existencia de riesgos a los que se expone la persona, no se entiende como ésta no se cuida para mantenerse en su peso dentro de la normalidad establecida por los expertos. De este modo, es fácil vincular la idea de que una persona obesa tiene falta de voluntad, carece de autocontrol, muestra dejadez, o es perezosa. La moralidad que transmiten los discursos sobre el control del peso corporal puede servir de herramienta para la exclusión o inclusión de las personas en el grupo de normales (EVANS et al., 2008). Estas actitudes influyen negativamente en la percepción que una persona tiene de sí misma afectando negativamente a su autoconcepto y autoestima (COMPANY, 2013; GIL, 2006; GOÑI; RODRIGUEZ, 2004).

Finalmente, al poner el énfasis en la persona se obvia los factores no sólo genéticos, sino también socioeconómicos y culturales que determinan el sobrepeso y la obesidad. Diferentes estudios muestran la existencia de asociación entre factores socioeconómicos, culturales y obesidad (AZAR et al., 2015; BAUM; RUHM, 2009; MARÍN et al., 2010; ORTIZ et al., 2006). La investigación ha mostrado diferencias culturales al evidenciar un menor riesgo de obesidad y sobrepeso en las personas de mayor nivel social y académico (AZAR et al., 2015; ROSKAM et al., 2009). Sin embargo, los discursos de la obesidad parecen obviar las influencias sociales y culturales, de tal manera que sobredimensionan la responsabilidad individual a través de la 
regulación que cada uno puede hacer en el equilibrio entre ingesta y consumo energético. Con ello la responsabilidad institucional y social sobre la obesidad queda diluida.

En este artículo se han mostrado inconsistencias en algunos de los reclamos que acompañan el discurso sobre la obesidad, pero también se han considerado los efectos negativos imprevistos que estos discursos pueden tener en la salud de las personas. Esto nos hace pensar en la necesidad de promover una perspectiva crítica sobre el discurso de la obesidad y en una manera distinta de concebir la salud, otorgando mayor importancia a los factores culturales y sociales. Sobre esta base cabría preguntarnos ¿hasta dónde debe presionar la sociedad a una persona sana con sobrepeso que práctica una dieta equilibrada, realiza actividad física regularmente y su peso no ha variado en años? Entendemos que esta presión nunca debería perjudicar la salud física, psíquica o social de las personas con sobrepeso u obesidad. En este artículo se cuestiona que la sociedad en su conjunto no debería asumir estos riesgos potenciales para la salud, derivados precisamente de la presión social ejercida para el control del peso corporal.

\section{REFERENCIAS}

AGENCIA ESPAÑOLA DE SEGURIDAD ALIMENTARIA Y CONSUMO. Estudio ALADINO 2013:

Estudio de Vigilancia del Crecimiento, Alimentación, Actividad Física, Desarrollo Infantil y Obesidad en España 2013. 2014. Disponible en: < http://www.ciberobn.es/media/434027/estudio aladino 2013.pdf >. Acceso en: 23 sept. 2016.

AGENCIA ESPAÑOLA DE SEGURIDAD ALIMENTARIA Y CONSUMO. Estrategia NAOS: Estrategia para la nutrición, actividad física y prevención de la obesidad. Disponible en: <http://www.aecosan. msssi.gob.es/AECOSAN/docs/documentos/nutricion/estrategianaos.pdf >. 2005. Acceso en: 23 sept. 2016.

AGENCIA ESPAÑOLA DE SEGURIDAD ALIMENTARIA Y CONSUMO. Estudio de prevalencia de la obesidad infantil "ALADINO". Disponible en: $<$ http://www.aecosan.msssi.gob.es/AECOSAN/docs/ documentos/nutricion/observatorio/estudio ALADINO 2011.pdf >. 2013. Acceso en: 23 sept. 2016.

ALEMANY, Marià. Mecanismos de control del peso corporal. Revista de la Real Academia de Medicina de Cataluña, Barcelona, v. 18, n. 2, p. 44-49, 2003.

AZAR, Ariel et al. Determinantes individuales sociales y ambientales del sobrepeso y la obesidad adolescente en Chile. Revista Médica de Chile, Santiago, v. 143, p. 598-605, 2015.

BAUM, Charles; RUHM, Christopher. Age, socioeconomic status and obesity growth. Journal of Health Economic, New York, v. 28, p. 635-648, 2009.

BURT, Julia et al. Sleep and eating in childhood: a potential behavioral mechanism underlying the relationship between poor sleep and obesity. Sleep medicine, New York, v. 15, n. 1, p. 71-75, 2014.

CAMPOS, Paul. The obesity Myth. New York: Gotham Books, 2004.

CASAZZA, Katherine et al. Myths, Presumptions, and Facts about Obesity. New England Journal of Medicine, Massachusetts, v. 368, n. 5, p. 446-454, 2013. Disponible en: < http://www.nejm.org/doi/ full/10.1056/NEJMsa1208051 >. Acceso en:: 23 sept. 2016

COLE, Tim et al. Establishing a standard definition for child overweight and obesity worldwide: international survey. British Medical Journal, London, v. 320, p. 1240-1243, 2000. 
COLEMAN, Daniel. A historical perspective on leptin. Nature Medicine, New York, v. 16, p. 10971099, 2010.

COMISIÓN EUROPEA. Libro blanco sobre la obesidad. Bruselas: Comisión Europea, 2007.

COMPANY, Miguel. La influencia de los discursos sobre salud y obesidad en los escolares de secundaria obligatoria. Almería: Universidad de Almería, 2013.

CORRELLA, Dolores; PORTOLÉS, Olga. Avances en el conocimiento de las bases genéticas de la obesidad. Genética, Nutrición y Enfermedad. Madrid: Edimsa, 2008.

EVANS, John et al. Education, Disordered Eating and Obesity Discourse. Fat fabrications. Londres: Routledge, 2008.

FAROOQI, Sadaf; O'RAHILLY Stephen. Monogenic obesity in humans. Annual Review of Medicine, n. 56, p. 443-458, 2005.

FLEGAL, Katherine et al. Excess deaths associated with underweight, overweight, and obesity. Journal of the American Medical Association, Chicago, v. 293, n. 15, p. 1861-1867, 2005.

FLEGAL, Katherine et al. Cause-specific excess deaths associated with underweight, overweight, and obesity. Journal of the American Medical Association, v. 298, n. 17, p. 2028-2037, 2007.

FLEGAL, Katherine et al. Association of all-cause mortality with overweight and obesity using standard body mass index categories: A systematic review and meta-analysis. Journal of the American Medical Association, v. 309, n. 1, p. 71-82, 2013.

FOZ, Mario. ¿Es el sobrepeso nocivo para la salud? Revista Española de Obesidad, v. 6, n. 2, p. 62-65, 2008.

GARD, Michael. The end of the epidemic obesity. New York: Routledge, 2011.

GARD, Michael; WRIGHT, Jan. The obesity epidemic. Science, morality and ideology. Londres: Routledge, 2005.

GIL, Eugenia. Anorexia y bulimia en las mujeres: entre la inseguridad y la autonomía. Revista Crítica, v. 933, p. 82-84, 2006.

GOÑI, Alfredo; RODRIGUEZ, Alberto. Trastornos de la conducta alimentaria, práctica deportiva, y autoconcepto físico en adolescentes. Actas Españolas de Psiquiatría, v. 32, n. 1, p. 29-36, 2004.

GUALLAR-CASTILLÓN, Pilar et al. Magnitud y manejo del síndrome metabólico en España en 20082010: Estudio ENRICA. Revista Española de Cardiologia, v. 67, n. 5, p. 367-373, 2014.

HARSCH, lan et al. Leptin and ghrelin levels in patients with obstructive sleep apnoea: effect of CPAP treatment. European Respiratory Journal, v. 22, n. 2, p. 251-257, 2003.

HARWOOD, Valerie. Tehorizing Biopedagogies. In: WRIGHT, Jan ; HARWOOD, Valerie. Biopolitics and the "Obesity Epidemic" Goberning Bodies. New York: Routledge, 2009.

HERNÁNDEZ, Manuel et al. Curvas y tablas de crecimiento. Instituto de Investigación sobre Crecimiento y Desarrollo, Fundación Faustino Orbegozo. Madrid: Garsi, 1998.

INSTITUTO NACIONAL DE ESTADÍSTICA. Encuesta Europea de Salud. 2009. Disponible en: $<$ http://www.ine.es/dyngs/INEbase/es/operacion.tm?c=Estadistica C\&cid= $1254736176784 \&$ menu=resultados\&idp=1254735573175 > . Acesso en: 23 sept. 2016.

JARRIN, Denise; MCGRATH, Jennifer; POIRIER, Paul. Autonomic Dysfunction: A Possible Pathophysiological Pathway Underlying the Association Between Sleep and Obesity in Children At- 
Risk for Obesity. Journal of youth and adolescence, v. 44, n. 2, p. 285-297, 2015.

LÓPEZ, Ángeles. Epidemia de obesidad en Europa. Madrid: El Mundo. 2015. Disponible en: < http:// www.elmundo.es/salud/2015/05/06/55490221ca4741b84a8b4585.html >. Acceso en: 25 jun. 2015.

LÓPEZ DE LA TORRE, Martín; BELLIDO, Diego. El acoso social del obeso. Revista Española de Obesidad, v. 6, n. 5, p. 235-236, 2008.

LUGILDE, Anxo. La xunta retira la custodia de un niño con sobrepeso a sus padres. Barcelona: La Vanguardia. 2009. Disponible en: < http://www.lavanguardia.com/vida/20091030/53814039487/laxunta-retira-la-custodia-de-un-nino-con-sobrepeso-a-sus-padres.html >. Acceso en: 25 jun. 2015.

MARÍN, Ana et al. Prevalencia de obesidad en inmigrantes en Madrid. Medicina Clínica, v. 134, p. 483-485, 2010.

MINISTERIO DE SANIDAD, SERVICIOS SOCIALES E IGUALDAD. Encuesta Nacional de Salud 2006. 2007. Disponible en: < http://www.msps.es/estadEstudios/estadisticas/encuestaNacional/ encuesta2006.html>. Acceso en: 15 ene. 2015

MINISTERIO DE SANIDAD, SERVICIOS SOCIALES E IGUALDAD. Encuesta Nacional de Salud 2012. 2013. Disponible en: < http://www.msssi.gob.es/estadEstudios/estadisticas/encuestaNacional/ encuesta2011.html.> Acceso en: 15 ene. 2015.

MORENO, Basilio; MONEREO, Susana; ÁLVAREZ, Julia. La obesidad: La epidemia del siglo XXI. Madrid: Díaz de Santos, 2000.

O'BRIEN, James et al. Body mass index is independently associated with hospital mortality in mechanically ventilated adults with acute lung injury. Critical Care Medicine, v. 34, p. 738-744, 2006.

OGDEN, Cynthia; CARROLL, Margaret; FLEGAL, Katherine. Prevalence of obesity in the united states. Journal of the American Medical Association, v. 312, n. 2, p. 189-190, 2014. Disponible en: < http://dx.doi.org/10.1001/jama.2014.6228 >. Acceso en: 17 mar. 2015

OGDEN, Cynthia et al. Prevalence of childhood and adult obesity in the united states, 2011-2012. Journal of the American Medical Association, v. 311, n. 8, p. 806-814, 2014. Disponible en: < http:// dx.doi.org/10.1001/jama.2014.732 >. Acceso en: 17 mar. 2015.

OLIVER, J Eric. Fat politics: The real story behing America's obesity epidemic. Oxford: Oxford University Press, 2006.

OREOPOULOS, Antigone et al. Body mass index and mortality in heart failure: a meta-analysis. American Heart Journal, v. 153, n. 1, p. 13-22, 2008.

ORGANIZACIÓN MUNDIAL DE LA SALUD. Estrategia global sobre dieta, actividad física y salud. Ginebra: OMS 2004.

ORGANIZACIÓN MUNDIAL DE LA SALUD. Nota informativa no 311: Obesidad y sobrepeso. Ginebra, 2015. Disponible en: < http://www.who.int/mediacentre/factsheets/fs311/es/ >. Accesso: 3 feb.2015.

ORTEGA, Francisco et al. The intriguing metabolically healthy but obese phenotype: cardiovascular prognosis and role of fitness. European Heart Journal, v. 34, p. 394-397, 2013.

ORTIZ, Rocio et al. Determinantes sociales de sobrepeso y obesidad en España 2006. Medicina Clínica, v. 17, n. 15, p. 478-684, 2006.

ROMERO-CORRAL, Abel et al. Association of bodyweight with total mortality and with cardiovascular events in coronary artery disease: a systematic review of cohort studies. The Lancet, v. 368, p. 666678, 2006. 
ROSKAM, Albert et al. Comparative appraisal of educational inequalities in overweight and obesity among adults in 19 European countries. International Journal of Epidemiology, v. 39, p. 392-404, 2009.

SERRA, Lluís et al. Obesidad infantil y juvenil en España. Resultados del estudio EnKid. Medicina Clínica, v. 121, n. 19, p. 725-732, 2003.

SEVILLANO, Elena. Europa se enfrenta a una epidemia de obesidad en 2030. Madrid: El País. 2015. Disponible en: < http://elpais.com/elpais/2015/05/06/ciencia/1430935011 546446.html >. Acceso en: 25 jun. 2015.

USHMA, Neill. Leaping for leptin: the 2010 Albert Lasker Basic Medical Research Award goes to Douglas Coleman and Jeffrey M. Friedman. The Journal of Clinical Investigation, v. 120, n. 10, p. 3413-3418. 2010. 


\section{Apoyo:}

Ministerio Español de Economía y Competitividad, a través del proyecto de investigación titulado

"Imagen corporal y ejercicio fisico en la adolescencia: un estudio longitudinal" (Ref. DEP2014-57228-R). 